\title{
A Simple Autofocus Technique for Aberration Corrected TEM
}

\author{
G. Möbus \\ Dept. Engineering Materials, University of Sheffield, Mappin Street, Sheffield S1 3JD, UK
}

Alignment of objective aberration correctors [1] is now routinely achieved by automatic evaluation of ring patterns in diffractograms. To achieve sufficient sensitivity for the measurement of multiple aberration coefficients, an underfocused near-stigmatic imaging condition is required [2]. Near Gaussian focus the lack of both spherical aberration and defocus phase shifts leads to vanishing diffractogram features for weak-phase objects. Focusing criteria for $\mathrm{z}=0$ in the past have therefore been based on "minimum contrast" or on maximum contrast at Scherzer focus minus a calculated difference of 1 Scherzer unit.

Another approach is via deliberate high astigmatism [3,4]. A diffractogram with astigmatism amplitude larger than the mean focus, $\Delta \mathrm{z}>\mathrm{z}_{\text {mean }}$, shows hyperbolic features with the central black fringes running along the hyperbolic main axes. For a weak-phase contrast transfer function we have:

$\operatorname{CTF}(k, z)=\sin \left(\pi / 2 \lambda^{3} C_{s} k^{4}+\pi \lambda\left(z_{\text {mean }}+\Delta z / 2 \sin \left(2\left(\theta-\theta_{0}\right)\right)\right) k^{2}\right) ; \quad$ for $C_{s}=0$ we can find from $z_{\text {mean }}+\Delta z / 2 \sin \left(2\left(\theta-\theta_{0}\right)=0\right.$ the two roots for $\theta$ separated by $\delta \theta$, with a unique $\delta \theta=\pi / 2$ for $z_{\text {mean }}=0$.

The curvature of the central cross of black fringes indicates spherical aberration. Consequently the straightness and the perpendicular crossing of these fringes can be used as a criterion for vanishing defocus and spherical aberration. For practical use it is required that astigmatism can be changed to large amplitudes reversibly without change of mean focus. This is not normally fulfilled for TEMs, but works fine for the internal stigmators of the aberration correction system. This is confirmed in Fig 1 which shows two diffractograms recorded at zero focus for two largely different astigmatism values, using the Sheffield JEM 2200FS-AC (double-aberration corrected) with CEOS correctors $[5,6]$. While hyperbolic diffractograms are not eligible for standard imaging parameter metrology, automatic identification of the main axis cross can be easily implemented.

A second application of hyperbolic diffractograms is the evaluation of continuous contrast-transferfunctions (CTF) with focus via its interpretation as an azimuthal focal series. The conversion to a Cartesian $\operatorname{CTF}(\mathrm{k}, \mathrm{z})$ diagram requires non-equidistant resampling of radial sections [4], equivalent to Frank's contrast transfer nomograms (CTN) or Thon diagram [7,8], see Fig 1c. The horizontal straight black fringe at $\mathrm{z}=0$ and the symmetric appearance around it indicates again vanishing spherical aberration. Alternatively, access to experimental $\mathrm{CTF}(\mathrm{k}, \mathrm{z})$ data is via tilted carbon films [9]. Using a tomography holder able to tilt to $80^{\circ}$ in a $2 \mathrm{~mm}$ pole piece [10], Fig 2 shows the image intensity and its one-dimensional FFT corresponding to the CTN. This 1D-FFT is important for aligning focus for tomographic tilt series by shifting the minimum contrast fringe to the particle of interest within the field of view. A 2D-FFT would only result in a blurred diffractogram with no automatic ring evaluation possible. As the CTN refers to the carrier film, focusing of particles will still be problematic if they sit more than $5-10 \mathrm{~nm}$ elevated from the film, e.g. as part of a cluster touching the film elsewhere.

\section{References}

[1] M. Haider et al., Ultramicroscopy 75 (1998) 53.

[2] M. Haider et al., CEOS aberration corrector, user manual.

[3] G Möbus, Dissertation, University of Stuttgart, Germany, 1994.

[4] G Möbus et al., J. Electr. Micr. 46 (1997) 381-395. 
[5] G Möbus et al., Inst.Phys.Conf.Ser. 168 (2001) 27-30.

[6] J.L. Hutchison et al., Proceedings ICEM-15, Durban, SA, 3 (2002) 25-26.

[7] R.E. Burge, R.F. Scott, Optik 43 (1975) 503.

[8] F. Thon, In U.Valdre, ed., Electron Microscopy in Materials Science, Academic Press, New York, 1977.

[9] W. Krakow, K.H. Downing, B.M. Siegel, Optik 40 (1975) 1.

[10] X. Xu et al., Mater.Res.Soc.Symp.Proc. 928E (2007) 0982-KK02-04.

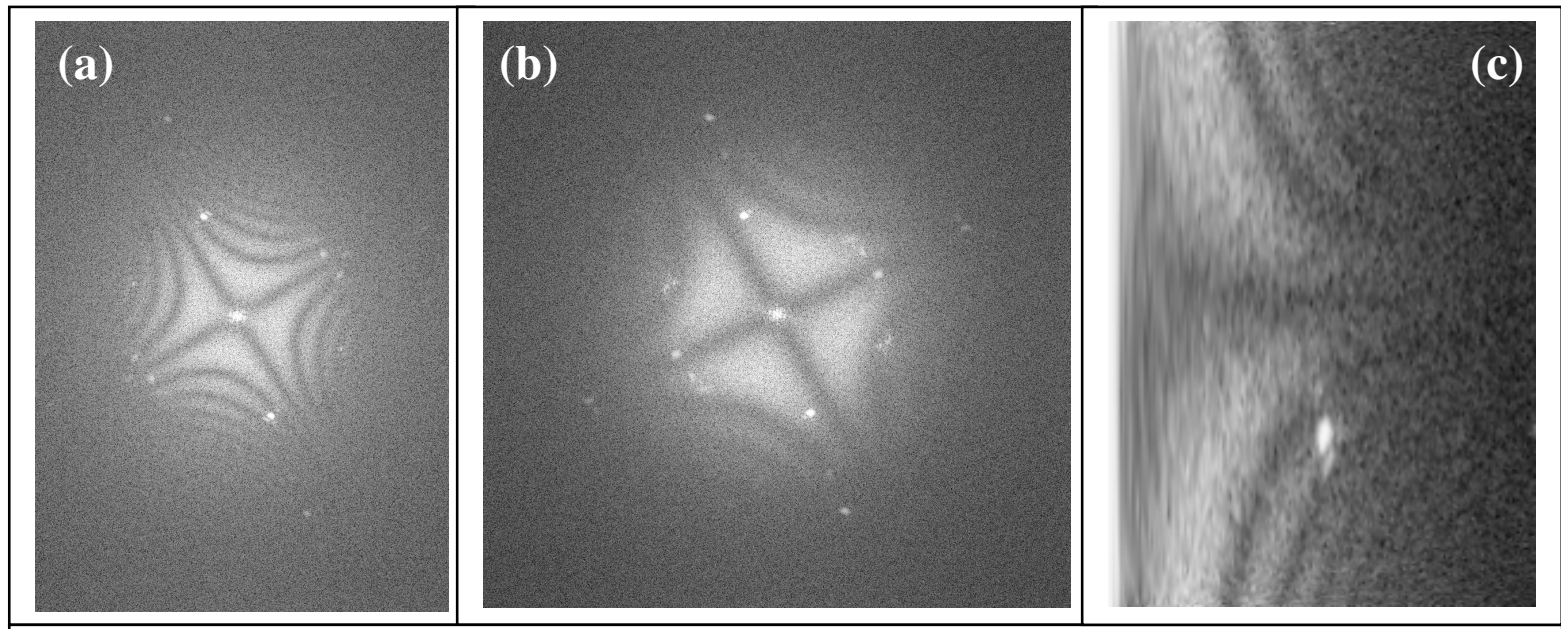

FIG. 1. FFT of image intensity of amorphous carbon film for $\mathrm{Cs}=\mathrm{z}=0$ (Gaussian focus) and strong astigmatism with $(\mathrm{a}, \mathrm{b})$ two different astigmatism amplitudes. (c) CTN (k,z) derived from (a) with $\mathrm{z}$ as vertical and $\mathrm{k}$ as horizontal coordinate axes.

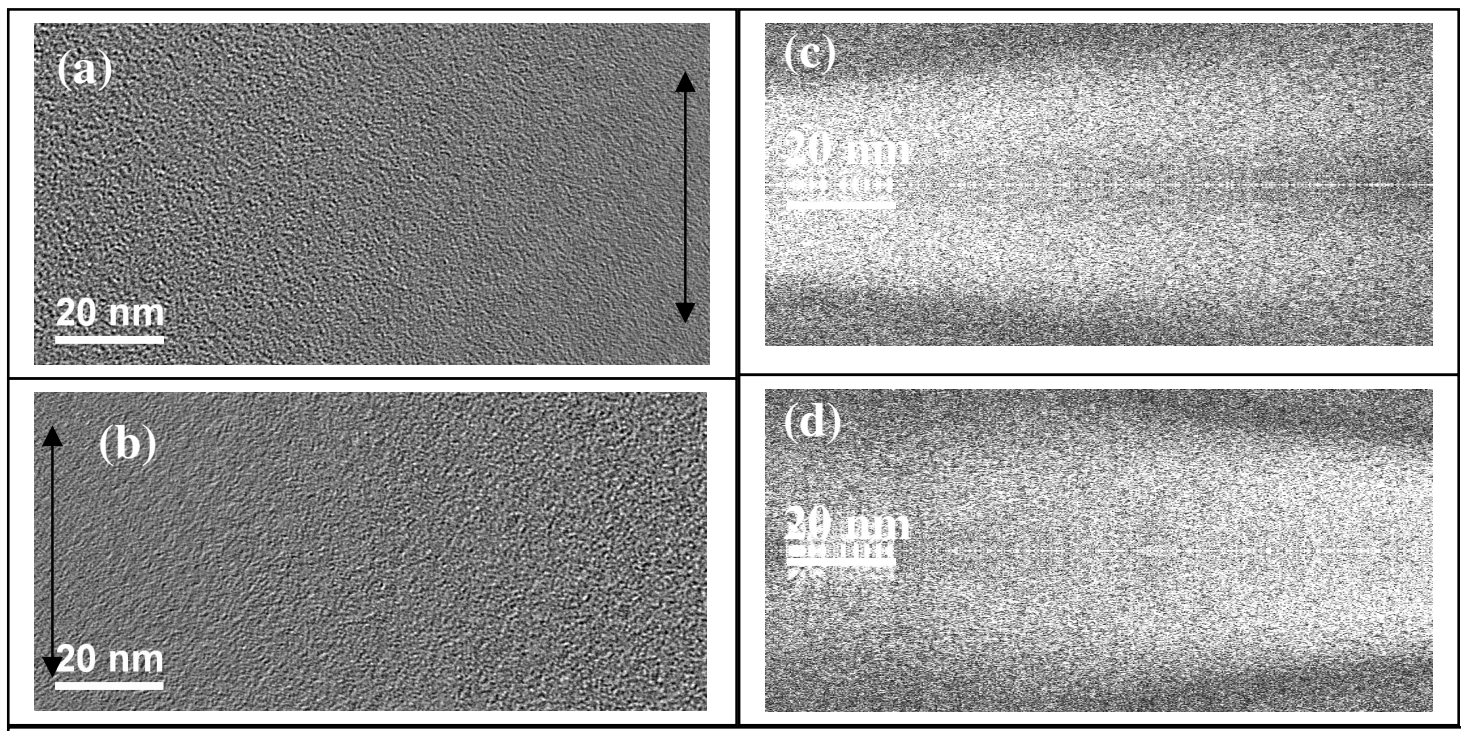

FIG. 2. (a-b): Images of amorphous carbon under $50^{\circ}$ inclination for two focus values shifting the minimum contrast region $(\leftarrow \rightarrow)$. $(\mathrm{c}, \mathrm{d}) \mathrm{CTN}(\mathrm{z}, \mathrm{k})$ via one-dim. FFT from $(\mathrm{a}, \mathrm{b})$ with $\mathrm{z}$ as horizontal and $\mathrm{k}$ as vertical coordinate. 